\title{
Retrospective analysis of sorafenib efficacy and safety in Chinese patients with high recurrence rate of post-hepatic carcinectomy
}

This article was published in the following Dove Press journal: OncoTargets and Therapy

Desheng Wang, ${ }^{1, *}$ Weridong Jia, ${ }^{2, *}$ Zhiming Wang, ${ }^{3}$ Tianfu Wen, ${ }^{4}$ Wei Ding, ${ }^{5}$ Feng Xia, ${ }^{6}$ Ling Zhang, ${ }^{7}$ Feixiang Wu, ${ }^{8}$ Tao Peng, ${ }^{9}$ Bin Liu, ${ }^{10}$ Cuncai Zhou, "' Qichang Zheng, ${ }^{12}$ Xiongying Miao, ${ }^{13}$ Junping Peng, ${ }^{14}$ Zhiyong Huang, ${ }^{15}$ Kefeng Dou'

'Department of Hepatobiliary Surgery, Xijing Hospital, Shaanxi, China; ${ }^{2}$ Department of Hepatobiliary Surgery, Anhui Provincial Hospital, Hefei, China; ${ }^{3}$ Department of Hepatobiliary Surgery, Xiangya Hospital, Changsha, China; ${ }^{4}$ Department of Hepatobiliary Surgery, West China Hospital, Chengdu, China; ${ }^{5}$ Department of Hepatobiliary Surgery, Cancer Hospital of Xinjiang, Urumqi, China; 'Department of Hepatobiliary Surgery, Southwest Hospital, Chongqing, China;

${ }^{7}$ Department of Hepatobiliary Surgery, Cancer Hospital of Henan, Zhengzhou, China;

${ }^{8}$ Department of Hepatobiliary Surgery, Cancer Hospital of Guangxi Medical University,

Nanning, China; ${ }^{\circ}$ Department of Hepatobiliary Surgery, The First Affiliated Hospital of Guangxi Medical University, Nanning, China;

${ }^{10}$ Department of Hepatobiliary Surgery, The First Affiliated Hospital of Kunming Medical University, Kunming, China; " Department of Hepatobiliary Surgery, Cancer Hospital of Jiangxi, Nanchang, China; ${ }^{12}$ Department of Hepatobiliary Surgery, Wuhan Union Hospital, Wuhan, China; ${ }^{13}$ Department of Hepatobiliary Surgery, The Second Affiliated Hospital of Xiangya, Changsha, China; ${ }^{14}$ Department of Hepatopancreatobiliary Surgery, Cancer Hospital of Sichuan, Chengdu, China;

${ }^{15}$ Department of Hepatobiliary Surgery, Wuhan Tongji Hospital, Wuhan, China

*These authors contributed equally to this work

Correspondence: Kefeng Dou

Department of Hepatobiliary Surgery, Xijing Hospital, The Fourth Military Medical

University, Changle West Road, NO.127, Xi'an,

Shaanxi, China

Tel +8613571890758

Fax +86 2984775255

Email gdwkgwx@fmmu.edu.cn
Background: There is no guideline recommendation for preventing hepatocellular carcinoma (HCC) recurrence after hepatic resection. Moreover, an unmet need exists on the effectiveness of sorafenib therapy in recurrent HCC.

Purpose: We therefore assessed the efficacy and safety of sorafenib in Chinese HCC patients with high risk of recurrence.

Patients and methods: Data were collected retrospectively from 15 Chinese research centers from January 1, 2012 to November 15, 2013, by chart reviews of patients with moderate-advanced HCC who received hepatic carcinectomy. The primary end point was recurrence-free survival rate at 1 year in patients with a high recurrence risk. Secondary end points included 1-year survival rate, time to recurrence and safety assessment.

Results: A total of 209 high-risk patients (sorafenib, $n=98$; control, $n=111$ ) who underwent carcinectomy were analyzed. There was no significant difference in the proportion of patients with recurrence-free survival at 1 year between the sorafenib and control $(70.43 \%$ vs 68.90\%: $\left.\chi^{2}=0.007, P=0.934\right)$. One-year survival rate was significantly higher with sorafenib than observed with control $\left(95.5 \%\right.$ vs $\left.83.35 \% ; \chi^{2}=7.441, P=0.006\right)$. Time to recurrence between sorafenib and control groups was similar. Incidences of all the adverse events (AEs) were similar in both the groups and transaminase elevation was most common in both groups $(20.37 \%$ vs $24.79 \%)$. Thrombocytopenia incidence was significantly lower with the sorafenib group than with control $(1.85 \%$ vs $9.40 \% ; P=0.015)$.

Conclusion: Sorafenib may be considered as a feasible option in the treatment of HCC recurrence.

Keywords: hepatocellular carcinoma, recurrence, sorafenib, survival, carcinectomy, Chinese

\section{Introduction}

Hepatic resection/carcinectomy is the preferred curative option among the multiple treatment modalities available for hepatocellular carcinoma (HCC). ${ }^{1,2}$ However, $\mathrm{HCC}$ recurrence remains the main detrimental factor in patients receiving hepatic resection, with a frequency as high as $70 \%-100 \% .^{3}$ In general, HCC recurs soon after or within 1 to 2 years after hepatic resection and liver transplantation and is associated with a dismal outcome and compromised long-term survival. ${ }^{4-6}$ Prevention, early detection of recurrence, and surgical management could further improve the survival rates.

The tyrosine kinase inhibitor, sorafenib (Nexavar ${ }^{\circledR}$; Bayer HealthCare, Wayne, NJ, USA), inhibits tumors angiogenesis through inhibition of vascular endothelial growth 
factor (VEGF) and platelet-derived growth factor (PDGF) signaling pathways, has demonstrated a significant prolongation in survival of patients with advanced-stage HCC. ${ }^{3,7}$ Being the only FDA-approved systemic chemotherapeutic agent, it is regarded as the gold standard in treating patients with advanced HCC. ${ }^{8}$ Lenvatinib has reported a promising clinical outcome with a median survival time of 13.6 months vs 12.3 months in sorafenib. ${ }^{9}$ Currently, the approval of lenvatinib as first-line therapy in unresectable hepatocellular carcinoma (HCC) is under FDA review. ${ }^{10}$ Regorafenib is the other multikinase inhibitor approved for the second line of therapy in advanced HCC. Treatment with sorafenib alone or in combination with mammalian target of rapamycin (mTOR) inhibitors has shown promising results in $\mathrm{HCC}$ recurrence. ${ }^{10-14}$ Available evidence from multiple studies showed that administration of sorafenib to patients with recurring HCC demonstrated better results compared with the control treatments. In a retrospective study by Saab et $\mathrm{al}^{15}$ recurrence rates of $12.5 \%$ were reported in patients who received adjuvant sorafenib therapy compared with $50 \%$ reported by the control population. However, the study did not provide important evidence regarding sorafenib efficacy in reducing the incidence of HCC recurrence and increasing the disease-free survival and overall survival for high-risk liver transplant recipients, hence further studies were warranted. A pilot study concluded sorafenib adjuvant therapy to be effective in reducing the risk of post-operative HCC relapse. A significantly higher time to recurrence was observed in the sorafenib group than the control group $(P=0.006)$. In addition, median recurrence-free survival in the sorafenib arm was prolonged by 8 months compared with the control group $(P=0.006)$, with a recurrence rate significantly lesser in the sorafenib group $(P=0.032) .{ }^{16}$ On the contrary, few studies have suggested limited effect of sorafenib treatment in HCC recurrence. ${ }^{11,17}$ A phase 3, double-blind, placebo-controlled study (STORM) of 1114 patients demonstrated no difference in median recurrence-free survival between the sorafenib group (33.3 months) and the placebo group (33.7 months; $P=0.26$ ) and concluded sorafenib adjuvant therapy to be ineffective in HCC following radical resection or ablation. ${ }^{18}$

On the contrary, many studies also indicate that efficacy of sorafenib treatment in recurrent HCC has not been sufficiently robust to inspire clinical use because of the observational study designs and limited sample sizes. ${ }^{19-21}$ Therefore, there is a lack of clarity on the effectiveness of sorafenib therapy in HCC recurrence. In addition, there is no universally accepted adjuvant therapy or consensus on the most apt treatment for preventing or treating HCC recurrence post hepatic resection. ${ }^{21,22}$ A recognized standard treatment for recurrence prevention in patients with a high recurrence risk after HCC excision remains to be elucidated. As a consequence, the prognosis for these patients is poor. ${ }^{23}$ Considering the scarce, inconsistent data, it is important to further explore the effectual benefit of sorafenib in treatment of HCC patients with recurrence. ${ }^{10}$ We therefore conducted this retrospective study to assess the efficacy and safety of sorafenib treatment in Chinese HCC patients with a high risk of recurrence.

\section{Materials and methods}

\section{Study design and patients}

We retrospectively reviewed data of patients with intermediate to advanced HCC who had received hepatic carcinectomy in 15 Chinese research centers from January 1, 2012 to November 15, 2013. Patients were eligible if they had histological confirmation of intermediate to advanced HCC (B-C stage of Barcelona Clinic Liver Cancer (BCLC) staging); received hepatocellular carcinectomy; A-B Child-Pugh classification and Eastern Cooperative Oncology Group Performance Status (ECOG) of $0-2$ before the surgical procedure; and a high possibility of disease recurrence (defined as patients having higher number of tumors, vascular invasion, and higher pathological staging). Recurrence rate of HCC is high; ranging from $8 \%$ to $15 \%$ and in our study, patients were considered to have a high recurrence rate if they met the following criteria: HCC rupture or invasion into adjacent organs (confirmed by intra-operative or post-operative pathology), positive margin (confirmed by post-operative pathology), nodal metastasis (confirmed by intra-operative or postoperative pathology); residual lesion as confirmed by post-operative digital subtract angiography (DSA); macroscopic/microscopic vascular and/or biliary cancer embolus (confirmed by intra-operative or post-operative pathology); three or more tumor lesions distributed over two or more hepatic segments (confirmed by preoperative imagology [CT, MRI or ultrasonography], intra-operative ultrasonography or post-operative pathology); deranged $\alpha$-fetoprotein AFP fails to return to normal range at 2 months after surgery (based on normal range in local laboratory). Sorafenib usage should either be $\leq 2$ months post procedure and a treatment duration $\geq 3$ months; or no use of sorafenib after the operation.

Patients were excluded from the study if they had initiated sorafenib $>2$ months after the surgical procedure; 
had relapse within 2 months after the surgical procedure as evident from radiographic examinations; had undergone anticancer treatment (except antiviral therapy) before the surgical procedure; had undergone organ transplantation; had another malignant visceral tumor; or were found to be HIV positive. The study protocols conformed to the ethical guidelines of the Declaration of Helsinki and were approved by the institutional review board (IRB) of each research center. The study methods were conducted in accordance with approved national and international guidelines.

\section{Study treatment}

We hypothesized that sorafenib improves 1-year progressionfree survival rate by $30 \%$. Considering this, as per the calculation of $95 \%$ study power and type I error of 0.05 , patient data for a minimum of 200 cases were to be collected within 6 months (100 cases in the sorafenib group and 100 cases in the control group), which would yield at least 150 patients with high-recurrence risk who can be evaluated in the study. Patients using sorafenib within 2-months post procedure and treatment duration of $\geq 3$ months were considered in the sorafenib group, whereas patients adopting treatment methods other than sorafenib such as hepatic transarterial chemoembolization (TACE) and systematic whole-body chemotherapy or immunotherapy ( $\alpha$-interferon) were considered in the control group. Sorafenib tosylate $(400 \mathrm{mg}$ ) was orally administered twice daily with low- or medium-fat food. Dosage adjustment was done according to recommendation in the instructions. Treatment was continued until the appearance of unacceptable toxicity, both radiological and clinical disease progression, or death. Patients received prescriptions for loperamide and ureacontaining ointments to treat instances of severe diarrhea and to prevent and treat skin reactions, respectively.

\section{Study outcomes}

Clinical and pathological data were collected and reviewed from patient medical records, and the most recent values were used before treatment initiation. The primary end point was recurrence-free survival rate at 1 year in patients with a high recurrence risk. The secondary end point was 1-year survival rate and time to recurrence. Safety was assessed by analyzing adverse events (AEs), which were graded according to the National Cancer Institute Common Terminology Criteria for Adverse Events v4.0 (NCI-CTCAE v4.0). ${ }^{38}$ Patients were observed from the time of carcinectomy until death, loss to follow-up, or end of the study.

Recurrence-free survival rate at 1 year was defined as the ratio of patients with no relapse within the duration from the date of carcinectomy until the date of completion of a year. One-year survival rate was defined as the ratio of patients who were still alive within the duration from the date of carcinectomy until the date of completion of 1 year. Time to recurrence refers to the time (number of days) from hepatocellular carcinectomy to the first recurrence of disease, including both intrahepatic and extrahepatic recurrence. As we evaluated the recurrence rate and recurrence free survival for 1-year after hepatic carcinectomy, our study includes only early recurrence type after the resection.

\section{Statistical analysis}

Descriptive statistical methods were used to analyze baseline characteristics of patients. Continuous variables are summarized as mean \pm standard deviation (SD), median values, and ranges, whereas categorical variables are presented as absolute and relative frequencies. Baseline characteristics and postoperative staging and initial signs in the sorafenib and control groups using $\chi^{2}$ test and Wilcoxon rank sum test. Survival, time to progression (TTP), and duration of sorafenib treatment were estimated using the Kaplan-Meier method and compared by the log-rank test for univariate analysis. Cox proportional hazards regression model was used to determine high recurrence risk factor and preoperative patient features and to evaluate the associations between HCC, post-operative pathological staging, and recurrence-free survival rate. Hazard ratios (HRs) and two-sided 95\% confidence intervals (CIs) were derived from step-wise Cox proportional hazard regression model. Effect of all potential prognostic factors were determined using ANOVA. Wald inspection was used for determining the correlation. Adverse events were summarized according to the systematic organ classification after MedDRA coding and preferred terms. All $P$-values were twosided and were considered significant if $<0.05$. The statistical analysis of the collected data was performed using SAS (Statistical Analysis System) statistical software for Windows v. 9.1.3 (SAS Institute Inc., Cary, NC, USA).

\section{Results}

\section{Patient demographics and clinical characteristics}

A total of 209 patients with high recurrence risk who underwent $\mathrm{HCC}$ at 15 centers were analyzed. Of these patients, 98 were included in the sorafenib group and 111 were included in the control group. Case distributions at all the centers are presented in Table S1. The baseline characteristics of patients are presented in Table 1. The 
mean age of patients was significantly higher in the control group compared with the sorafenib group $(52.73 \pm 10.87$ years vs $48.57 \pm 12.61$ years, $P=0.012$ ), with a male predominance in both the groups. A significant difference was observed in BCLC staging in both the groups (sorafenib vs control: stage $\mathrm{B}, 54.08 \%$ vs $69.37 \%, P=0.036$; stage $\mathrm{C}$, $44.90 \%$ vs $28.82 \%, P=0.036)$. There were no other differences in terms of follow-up duration, Child-Pugh liver function status, or any other clinical parameter between these groups. Preoperative tumors load in both the groups are presented in Table S2. Post-operative observations of the patients are detailed in Tables 2 and 3. More than $80 \%$ of the patients initiated sorafenib therapy within 1-8 weeks after the operation.

\section{Recurrence free survival at I-year}

All the 98 patients in the sorafenib group and 111 patients in the control group were included in survival analysis to determine the recurrence free survival rates and recurrence time. During the 1-year follow-up, 28 and 34 cases of recurrence or death occurred in the sorafenib group and control groups, respectively. The recurrence free survival at 1-year was nonsignificantly higher with sorafenib treatment compared to the control group (sorafenib: 70.43\%; control: 68.90\%; $\chi^{2}=0.007, P=0.934$ ), Figure 1 . Further cox regression analysis revealed that the treatment factors (HR 0.972, 95\% CI $0.589,1.607 ; P=0.913$ ), gender (HR 0.970, 95\% CI 0.442 , 2.130; $P=0.939$ ) and preoperative BCLC staging (HR 1.073, $95 \%$ CI $0.695,1.658 ; P=0.749$ ) had no significant impact on the recurrence free survival at 1 year.

\section{One-year survival rate and time to recurrence}

There were four cases of death in the sorafenib group and 18 cases in the control group. One-year survival rate was significantly higher in the sorafenib group compared to the control group ( $95.56 \%$ vs $83.35 \% ; \chi^{2}=7.441, P=0.006$, Figure 2$)$. As per Cox regression analysis for survival, between-the-group difference was significant (HR, 0.279; 95\% CI, 0.092-0.850; $P=0.025$; and BCLC staging difference was non-significant (HR, 0.640; 95\% CI, 0.240-1.710; $P=0.374$ ). During the 1-year follow-up, the mean recurrence time in HCC patients after treatment with sorafenib and control was not significantly different (196.75 \pm 152.50 days vs $232.87 \pm 173.15$, Wilcoxon rank statistic: $0.792, P=0.428$ ), Table 4 . Treatment-related factors (HR, 0.255, 95\% CI, 0.086-0.756, $P=0.014$ ) showed significant association with 1-year survival rate. However, gender
(HR, 1.855, 95\% CI, 0.627-5.486, $P=0.264$ ) and preoperative BCLC staging (HR, 0.694, 95\% CI, 0.285-1.688, $P=0.421$ ) demonstrated a non-significant association with time to recurrence as per Cox regression analysis (HR, 1.089, 95\% CI, $0.721-1.643, P=0.686$; and $1.116,0.801-1.556, P=0.516)$. The difference in survival rates was non-significant. Table 5 presents the overall 1-year recurrence rate, 1-year recurrence after excluding patients with deaths and recurrence in BCLC stage $\mathrm{B}$ and $\mathrm{C}$ patients after treatment with sorafenib and control. Recurrence rates for all the evaluations were higher after sorafenib treatment; however, the difference was non-significant.

\section{Safety events}

Commonly observed AEs in both the groups are listed in Table 6. A total of 104 AEs was observed in the sorafenib group and 116 in the control group. The incidence of all the AEs was similar in both the groups except for thrombocytopenia, which was significantly lesser in the sorafenib group (1.85\%) than the control group $(9.40 \%$; $P=0.015)$. In addition, transaminase elevation had the highest frequency in both the groups $(20.37 \%$ vs $24.79 \% ; P=0.429)$. There were three events of hand-foot skin reaction in the sorafenib group, whereas no such reaction was observed in the control group.

\section{Discussion}

Although an optimal treatment strategy, hepatocellular carcinectomy and resection has a major drawback of relapse/recurrence in majority of the cases. Effective results obtained with sorafenib in the treatment of HCC have led to exploration of the drug's application in recurrence. ${ }^{10,11}$ In addition, recommendation on the posttransplant use of sorafenib therapy in HCC recurrence remains to be elucidated. HCC recurrence either after resection or transplantation portrays poor prognosis of patients. $^{24}$ This large, multicenter, retrospective analysis evaluated the effect of sorafenib in 209 patients with a high risk of recurrence post-hepatic carcinectomy.

Sorafenib has shown efficacy in treating recurrent $\mathrm{HCC}$ after liver transplantation (LT) and hepatic resection alone as adjuvant therapy and by demonstrating a synergistic effect in combination with sirolimus. ${ }^{10,11,25}$ Nonetheless, the response rate and time to tumor progression in patients treated with sorafenib for recurrent $\mathrm{HCC}$ after transplantation were found to be comparable to HCC patients without transplantation. ${ }^{19,26}$ A pilot study by Wang et $\mathrm{al}^{16}$ (2014) demonstrated a significantly higher time to recurrence in the sorafenib group than in the control group $(21.45 \pm 1.98$ vs $13.44 \pm 2.66$ 
Table I Characteristics of the study population

\begin{tabular}{|c|c|c|c|}
\hline Parameters & Sorafenib $(n=98)$ & Control $(n=I I I)$ & $P$-value \\
\hline Age, median (IQR) & $46.5(15-77)$ & $55(30-79)$ & 0.012 \\
\hline Follow-up, mean days (SD) & $732.63(421.91)$ & $826.52(463.00)$ & 0.143 \\
\hline \multicolumn{4}{|l|}{ Gender, n (\%) } \\
\hline Male & $86(87.76)$ & $100(90.09)$ & 0.59 \\
\hline Female & $12(12.24)$ & $11(19.91)$ & \\
\hline \multicolumn{4}{|l|}{ BCLC staging, (\%) } \\
\hline Stage B & $53(54.08)$ & $77(69.37)$ & 0.036 \\
\hline Stage C & $44(44.90)$ & $32(28.83)$ & \\
\hline Unable to evaluate & $\mathrm{I}(1.02)$ & $2(1.80)$ & \\
\hline \multicolumn{4}{|l|}{ Child-Pugh overall score, n (\%) } \\
\hline Stage A & 87 (88.78) & $90(81.08)$ & 0.331 \\
\hline Stage B & $10(10.20)$ & $18(16.22)$ & \\
\hline Stage C & $0(0.0)$ & $2(1.80)$ & \\
\hline Unable to evaluate & I (I.02) & I $(0.90)$ & \\
\hline \multicolumn{4}{|l|}{ Surgical margin, $\mathbf{n}(\%)$} \\
\hline R0 excision & $90(92.78)$ & $107(96.40)$ & 0.245 \\
\hline RI excision & $7(7.22)$ & $4(3.60)$ & \\
\hline Missing & $\mathrm{I}(\mathrm{I} .02)$ & $0(0.0)$ & \\
\hline \multicolumn{4}{|l|}{ Blood vessel and bile duct invasion, $n$ (\%) } \\
\hline None & $56(57.73)$ & $60(54.55)$ & 0.645 \\
\hline Existing & $41(42.27)$ & $50(45.45)$ & \\
\hline Missing & I (1.02) & I $(0.90)$ & \\
\hline \multicolumn{4}{|l|}{ Lymphatic metastasis, n (\%) } \\
\hline None & $96(97.96)$ & $108(98.18)$ & 1.000 \\
\hline Existing & $2(2.04)$ & $2(1.82)$ & \\
\hline Missing & $0(0.0)$ & I $(0.90)$ & \\
\hline \multicolumn{4}{|l|}{ HCC initial diagnosis, $n$ (\%) } \\
\hline Serum tumor markers & $31(31.63)$ & $34(30.63)$ & 0.342 \\
\hline Imagological examination & $58(59.18)$ & $72(64.86)$ & \\
\hline Pathological examination & $8(8.16)$ & $3(2.70)$ & \\
\hline Unknown & $0(0.00)$ & I $(0.90)$ & \\
\hline Others & $\mathrm{I}(1.02)$ & I $(0.90)$ & \\
\hline AFP (IU/mL), mean (SD) & $994.69(1817.97)$ & $1071.79(1983.35)$ & 0.958 \\
\hline \multicolumn{4}{|l|}{ AFP clinical assessment, $\mathbf{n}(\%)$} \\
\hline Normal & $26(31.33)$ & $29(29.59)$ & 0.865 \\
\hline Abnormal - no clinical significance & $3(3.6 I)$ & $5(5.10)$ & \\
\hline Abnormal - clinical significance & $54(65.06)$ & $64(65.31)$ & \\
\hline Unknown/not checked & $0(0.0)$ & $13(\mid 1.7)$ & \\
\hline \multicolumn{4}{|l|}{ AFP, n (\%) } \\
\hline Normal & $30(30.61)$ & $23(20.72)$ & 0.497 \\
\hline Abnormal - no clinical significance & $3(3.06)$ & $3(2.70)$ & \\
\hline Abnormal - clinical significance & $14(14.28)$ & $18(16.21)$ & \\
\hline Unknown/not checked & $0(52.04)$ & $67(60.36)$ & \\
\hline Max tumor diameter, mean $\mathrm{cm}(\mathrm{SD})$ & $7.91(3.42)$ & $7.12(3.21)$ & 0.075 \\
\hline
\end{tabular}

(Continued) 
Table I (Continued).

\begin{tabular}{|c|c|c|c|}
\hline Parameters & Sorafenib $(n=98)$ & Control $(n=I I I)$ & P-value \\
\hline Length of the sum, mean (SD) & $9.26(4.20)$ & $8.46(4.86)$ & 0.021 \\
\hline \multicolumn{4}{|l|}{ Pre-operative ECOG status, n (\%) } \\
\hline 0 & $54(55.67)$ & $71(63.96)$ & 0.173 \\
\hline I & $42(42.30)$ & $36(32.43)$ & \\
\hline 2 & I (I.02) & $4(3.60)$ & \\
\hline Unknown/missing & I (I.02) & $0(0.0)$ & \\
\hline
\end{tabular}

Table 2 Post-operative (before taking medicine) pathological grading and stage

\begin{tabular}{|c|c|c|c|}
\hline Parameters & $\begin{array}{l}\text { Sorafenib } \\
(n=98)\end{array}$ & $\begin{array}{l}\text { Control } \\
(n=I I I)\end{array}$ & $P$-value \\
\hline Post-operative BCLC staging, n (\%) & $\mathrm{n}=75$ & $n=79$ & \\
\hline Stage A & $3(4.00)$ & $2(2.56)$ & 0.036 \\
\hline Stage B & $25(38.89)$ & $39(50.00)$ & \\
\hline Stage C & $4 \mid(27.78)$ & $37(50.00)$ & \\
\hline Stage D & $2(I I . I I)$ & $0(0.00)$ & \\
\hline Unable to evaluate & $4(5.33)$ & $0(0.0)$ & \\
\hline Post-operative staging T, n (\%) & $n=56$ & $n=63$ & \\
\hline I & $10(17.86)$ & $10(15.63)$ & 0.413 \\
\hline 2 & $14(25.00)$ & $2 I(32.8 I)$ & \\
\hline 3 & $30(53.57)$ & $27(42.19)$ & \\
\hline 4 & $2(3.57)$ & $6(9.38)$ & \\
\hline \multicolumn{4}{|l|}{ Post-operative staging N, n (\%) } \\
\hline 0 & $53(100.00)$ & $4(100.00)$ & 0.341 \\
\hline I & $3(5.36)$ & I (I.59) & \\
\hline \multicolumn{4}{|l|}{ Post-operative staging $\mathbf{M}, \mathbf{n}(\%)$} \\
\hline 0 & $56(100.00)$ & $63(98.4 I)$ & \\
\hline 1 & $0(0.0)$ & $0(0.0)$ & \\
\hline \multicolumn{4}{|l|}{ Number of lesions, $\mathbf{n}(\%)$} \\
\hline 1 & $67(68.37)$ & $78(70.91)$ & 0.575 \\
\hline 2 & $13(13.27)$ & $14(12.73)$ & \\
\hline 3 & $6(6.12)$ & $10(9.09)$ & \\
\hline$>3$ & $12(12.24)$ & $8(7.27)$ & \\
\hline Post-operative diameter $(\mathrm{mm})$, mean (SD) & $7.83(3.48)$ & $7.29(3.33)$ & 0.253 \\
\hline Post-operative long diameter of sum( $\mathrm{mm}$ ), mean (SD) & $9.43(4.63)$ & $8.28(4.35)$ & 0.032 \\
\hline Blood vessel and bile duct invasion, $\mathbf{n}(\%)$ & $n=96$ & $n=110$ & \\
\hline No & $44(45.83)$ & $46(41.82)$ & 0.562 \\
\hline Yes & $52(54.17)$ & $64(58.18)$ & \\
\hline Post-operative lymph node metastasis, n (\%) & $n=97$ & $\mathrm{n}=97$ & \\
\hline No & $92(94.85)$ & $102(96.23)$ & 0.739 \\
\hline Yes & $5(5.15)$ & $4(3.77)$ & \\
\hline
\end{tabular}


Table 3 Comparison of post-operative initial signs at first interview between groups

\begin{tabular}{|c|c|c|c|}
\hline Items & $\begin{array}{l}\text { Sorafenib } \\
(n=98)\end{array}$ & $\begin{array}{l}\text { Control } \\
(n=I I I)\end{array}$ & $P$-value \\
\hline Post-operative ECOG, n (\%) & $(n=77)$ & $(n=46)$ & \\
\hline 0 & $26(33.77)$ & II (23.9I) & 0.2505 \\
\hline I & $40(51.95)$ & $21(45.65)$ & 0.4993 \\
\hline 2 & II (I4.29) & $14(30.43)$ & 0.0321 \\
\hline Post-operative Child Pugh score, n (\%) & $(n=96)$ & $(n=74)$ & \\
\hline A & $64(66.67)$ & $46(60.53)$ & 0.4097 \\
\hline B & II (II.46) & $12(15.79)$ & 0.4118 \\
\hline C & $\mathrm{I}(\mathrm{I} .04)$ & $0(0.00)$ & \\
\hline Unable to evaluate & $20(20.83)$ & $18(23.68)$ & 0.6578 \\
\hline Post-operative AFP (IU/mL), mean (SD) & $439.78(889.75)$ & $503.96(1689.83)$ & 0.170 \\
\hline Post-operative AFP evaluation, n (\%) & $(n=78)$ & $(n=73)$ & \\
\hline Normal & $19(24.36)$ & $20(27.40)$ & 0.6708 \\
\hline Abnormal - no clinical significance & $\mathrm{I}(\mathrm{I} .28)$ & I (I.37) & 0.9616 \\
\hline Abnormal - clinical significance & $24(30.77)$ & $18(24.66)$ & 0.4040 \\
\hline Unknown/not checked & $34(43.59)$ & $34(46.58)$ & $0.7 \mid 48$ \\
\hline Post-operative visual results, n (\%) & $(n=47)$ & $(n=32)$ & \\
\hline Intrahepatic recurrence & 7 (14.89) & $8(25.00)$ & 0.2637 \\
\hline Distant metastasis & $0(0.00)$ & I (3.13) & - \\
\hline No recurrence/metastasis & $37(78.72)$ & $22(68.75)$ & 0.3202 \\
\hline Unable to evaluate & $3(6.38)$ & I (3.13) & 0.5204 \\
\hline
\end{tabular}

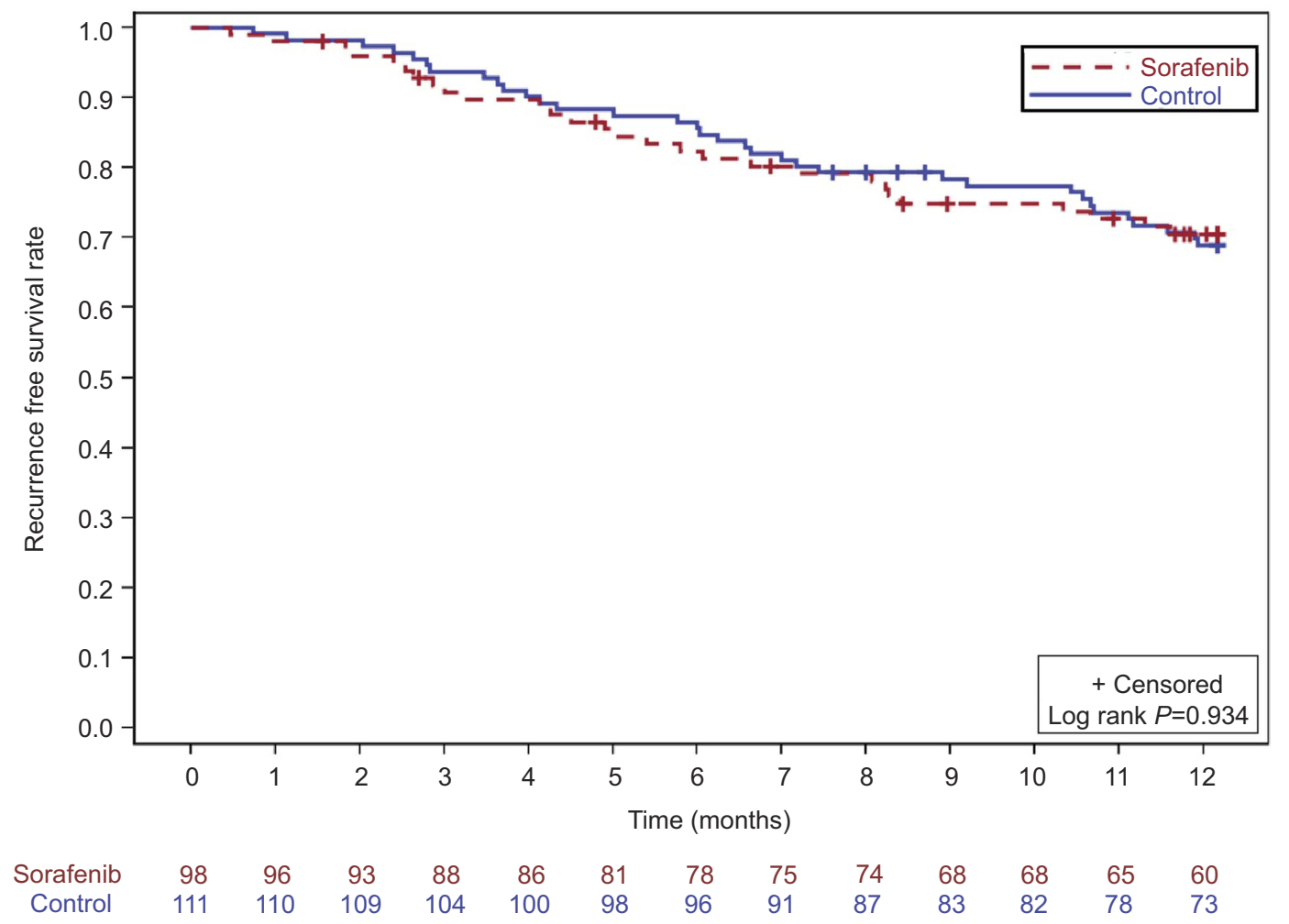

Figure I One-year recurrence-free survival rates. 


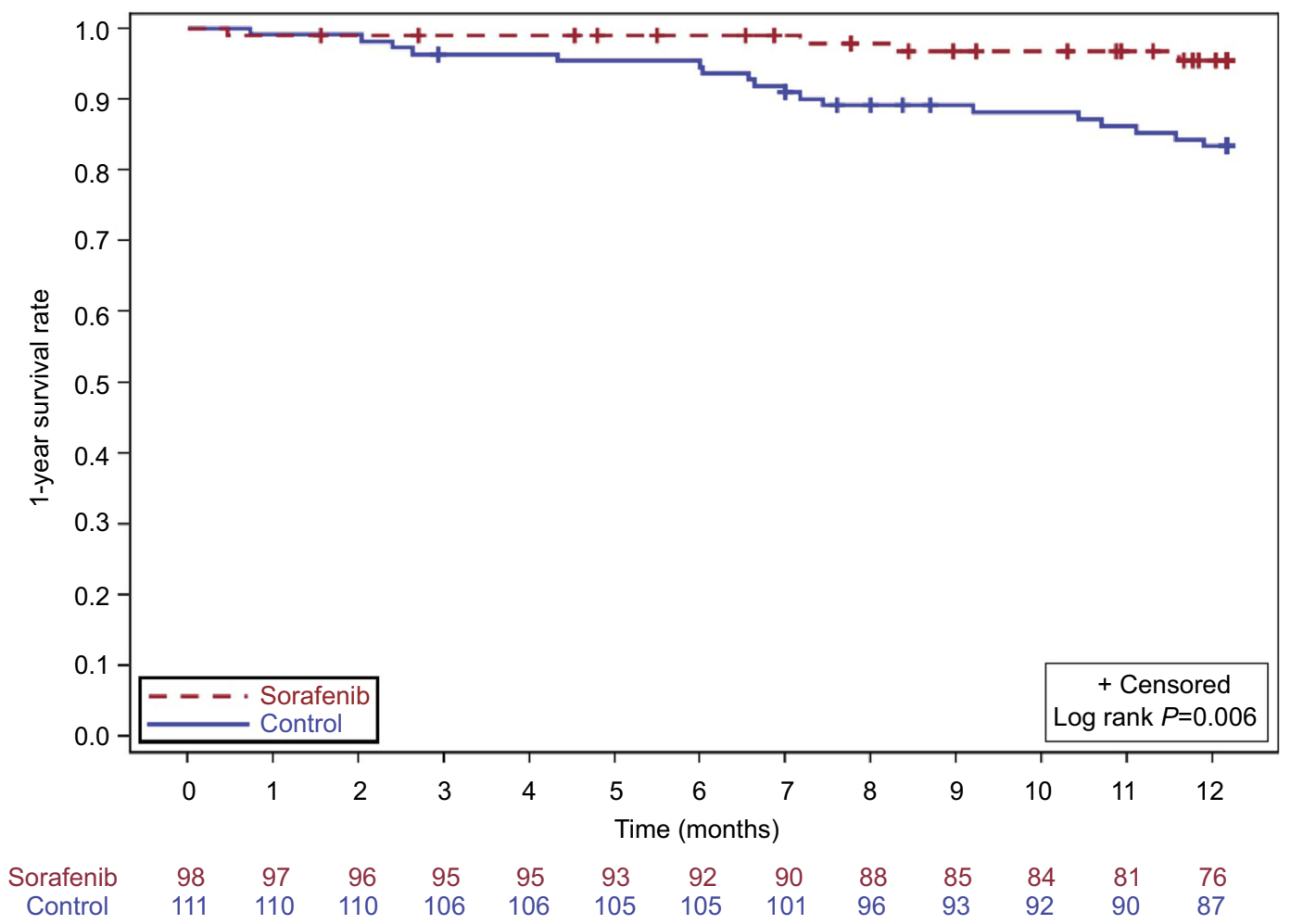

Figure 2 One-year survival rate.

Table $4 \mathrm{HCC}$ recurrence time (days)

\begin{tabular}{|l|l|l|l|l|}
\hline Index & Sorafenib & Control & Statistics & $P$-value \\
\hline N (missing) & $36(0)$ & $31(0)$ & 0.792 & 0.428 \\
Mean (SD) & $196.75(152.50)$ & $232.87(173.15)$ & Wilcoxon rank sum test \\
Median & 149.00 & 173.00 & - & - \\
QI, Q3 & $88.00,251.50$ & $85.00,358.00$ & - & - \\
Min, $\max$ & $27.00,789.00$ & $34.00,825.00$ & - & - \\
\hline
\end{tabular}

months, respectively; $P=0.006$ ), with a lesser recurrence rate in the sorafenib group than the control group (29.4\% vs $70.7 \%$; $P=0.032$ ). Adjuvant use of sorafenib was found to be safe and decreased the risk of HCC recurrence in high-risk LT recipients, with a disease-free survival rate of $100 \%$ in the sorafenib group. ${ }^{27}$ Similar beneficial effects of sorafenib in HCC recurrence post liver resection and transplantation have been reported in the literature. ${ }^{10,11,20,28,29}$ Of note, the International Consensus Conference for HCC recommends that HCC recurrence post liver transplantation may be treated with surgery for resectable lesions or with sorafenib for unresectable lesions. ${ }^{30}$ In a retrospective analysis, $\mathrm{Kim}_{\mathrm{im}} \mathrm{al}^{31}$ concluded that sorafenib had better survival benefit than other treatments in patients with extrahepatic spread and massive/infiltrative intrahepatic tumors. These outcomes indicate that sorafenib might be an important therapeutic modality in treating patients with extrahepatic recurrence. Furthermore, in a case-control study by Sposito et al, ${ }^{32}$ sorafenib demonstrated significantly better results than best medical care regimens in patients with $\mathrm{HCC}$ recurrence (median patient survival from recurrence: 21.3 months vs 11.8 months, $\mathrm{HR}=5.2, P=0.0009$ ). Moreover, multivariate analysis revealed sorafenib treatment to be the only factor associated with survival (HR, 4.0, $P=0.0325)$ with no serious AEs.

In the present study, we found that recurrence-free survival rate at 1 year was not significantly different between the two groups $(P=0.934)$. A similar study by Saab et $\mathrm{al}^{15}$ demonstrated significant increase compared to control in relatively smaller sample size. This difference, may be due to the high patient heterogeneity in terms of disease severity and progression. Similarly, time to relapse was non-significant between the two groups $(P=0.701)$. However, 1-year survival rate was 
Table 5 One-year recurrence rates



Table 6 Adverse events occurrence rates between the sorafenib and control groups

\begin{tabular}{|c|c|c|c|c|}
\hline Adverse events & Sorafenib, n (\%) & Control, n (\%) & Chi-square & $P$-value \\
\hline Diarrhea & $7(6.48)$ & $2(1.7 I)$ & 3.331 & 0.068 \\
\hline Fever & $5(5.56)$ & $5(4.27)$ & 0.199 & 0.656 \\
\hline Anemia & I (0.93) & $3(2.56)$ & 0.863 & 0.671 \\
\hline Thrombocytopenia & $2(1.85)$ & $10(9.40)$ & 5.880 & $0.015^{*}$ \\
\hline Nausea & I (0.93) & $3(2.56)$ & 0.180 & 0.671 \\
\hline Transaminase elevation & $20(20.37)$ & $28(24.79)$ & 0.625 & 0.429 \\
\hline Vomiting & I $(0.93)$ & $3(2.56)$ & 0.863 & 0.671 \\
\hline Leucocyte rises & $9(9.26)$ & $6(5.13)$ & $1.45 \mathrm{I}$ & 0.228 \\
\hline Increase in neutrophils & $8(8.33)$ & $7(5.98)$ & 0.470 & 0.493 \\
\hline Decrease in lymphocytes & $4(3.70)$ & I $(0.85)$ & 2.098 & 0.148 \\
\hline Decrease in albumin & I $(0.93)$ & $5(4.27)$ & 2.245 & 0.119 \\
\hline Hypokalemia & I $(0.93)$ & I $(0.85)$ & 0.262 & 0.609 \\
\hline Cough & $3(2.78)$ & $2(1.71)$ & 0.077 & 0.782 \\
\hline Rise in DBILI & $8(8.33)$ & $9(8.55)$ & 0.003 & 0.954 \\
\hline Total bilirubin & $6(6.48)$ & II (10.26) & 1.035 & 0.309 \\
\hline Abdominal distension & I (0.93) & I (0.85) & 0.003 & 0.955 \\
\hline HFSR & $3(2.78)$ & $0(0)$ & - & - \\
\hline
\end{tabular}

Note: *Clinically significant.

significantly higher in the sorafenib group than the control group $(P=0.006)$. This might be because recurrent tumors progressed at a slower pace after sorafenib treatment. Likewise, in a singlecenter retrospective study of 78 patients, sorafenib did not significantly prolong recurrence-free survival compared with the control group (11.7 vs 11.0 months, respectively; $P=0.702$ ), but it significantly prolonged the overall survival (32.4 vs 25.0 months; $P=0.046$ ). In patients with $\mathrm{HCC}$ post curative resection, sorafenib did not significantly reduce the recurrence rate compared with the control group $(67.7 \%$ vs $78.3 \% ; P=0.737)$, but it significantly reduced the mortality rate $(28.1 \%$ vs $60.9 \%$; $P=0.004) .{ }^{33}$ Sorafenib inhibits tumor proliferation and 
angiogenesis by acting on multiple pathways; ${ }^{34-36}$ however, tumor cell killing (autophagy) with sorafenib is speculated to be through the stress induced by sorafenib involvement in autophagy of the HCC cells. ${ }^{37}$ Therefore, our findings of significantly higher 1-year survival rate and non-significantly higher recurrence free survival at 1-year might be associated with the mechanisms of action, respectively.

Although better patient compliance and fewer AEs are associated with a smaller dose of sorafenib in patients with liver transplantation and liver resection having a high recurrence risk, we found that $400 \mathrm{mg}$ of sorafenib twice daily did not lead to significant toxicity. ${ }^{15,38}$ Moreover, the incidence of thrombocytopenia was significantly lower in the sorafenib group than the control group $(P=0.015)$. Incidences of all other AEs were similar in both the groups. Though the patients treated with sorafenib had a significantly higher overall survival, there was no significant evidence that showed sorafenib can prevent recurrence. Even though xenograft model animal studies have reported that sorafenib can effectively suppress postsurgical HCC recurrence and metastasis, ${ }^{39}$ there are no such convincing evidences in human trials. The current study has several limitations and, hence, the inferences should be drawn with caution. The retrospective nature of the study and non-randomization of the included patients and small sample size, is similar to previously reported studies and the findings may not be sufficient to inspire clinical use, however, this study proves efficacy and safety of sorafenib in Chinese patients with recurrent post hepatic carcinectomy and we tried to exclude the screening bias by including the control group patients who received carcinectomy within 6-months prior to the time when carcinectomy was initiated in the sorafenib group patients. Hence, future large-sized, randomized controlled studies are warranted to validate the findings and prove generalizability and clinical use of sorafenib. Overall, we advocate that treatment of recurrent HCC requires a multidisciplinary approach involving hepatologists, surgeons, radiologists, oncologists, and radiation oncologists. Improvement in treatment objectives and modification of the current recommendation will certainly benefit patients with a high recurrence risk.

In conclusion, Sorafenib significantly improved the 1-year survival rate in Chinese HCC patients with high risk of recurrence, though it did not increase the 1-year recurrence-free survival rate and time to recurrence. With a satisfactory safety and efficacy profile, it may be considered a feasible treatment option for recurrent HCC. However, further large, randomized controlled trials are needed to confirm the outcomes.

\section{Study protocol approval}

The protocol for the study was approved by the IRBs of the following Chinese hospitals: Xijing Hospital, Shaanxi; Anhui Provincial Hospital, Hefei; Xiangya Hospital, Changsha; West China Hospital, Chengdu; Cancer Hospital of Xinjiang, Urumqi; Southwest Hospital, Chongqing; Cancer Hospital of Henan, Zhengzhou; Cancer Hospital of Guangxi Medical University, Nanning; The First Affiliated Hospital of Guangxi Medical University, Nanning; The First Affiliated Hospital of Kunming Medical University, Kunming; Wuhan Union Hospital, Wuhan; The Second Affiliated Hospital of Xiangya, Changsha; Cancer Hospital of Sichuan, Chengdu; and Wuhan Tongji Hospital, Wuhan.

\section{Informed consent}

As this study was retrospective in nature the need of informed consent was waived off by the IRBs; however, all patient data were kept confidential to maintain patient anonymity.

\section{Acknowledgments}

The authors thank Dr Amit Bhat and Karan Sharma (Indegene Pvt. Ltd, Bangalore India) for providing medical writing support and technical assistance in the development of this manuscript, as funded by Bayer Healthcare Co. Ltd., China.

\section{Author contributions}

All authors made substantial contributions to conception and design, acquisition of data, or analysis and interpretation of data; took part in drafting the article or revising it critically for important intellectual content; gave final approval of the version to be published; and agree to be accountable for all aspects of the work.

\section{Disclosure}

The authors report no conflicts of interest in this work.

\section{References}

1. Park J-W, Chen M, Colombo M, et al. Global patterns of hepatocellular carcinoma management from diagnosis to death: the BRIDGE study. Liver Int Off J Int Assoc Study Liver. 2015;35:2155-2166. doi:10.1111/liv.12818

2. Schwartz M, Roayaie S, Konstadoulakis M. Strategies for the management of hepatocellular carcinoma. Nat Clin Pract Oncol. 2007;4:424-432. doi:10.1038/ncponc0844

3. Saraswat VA, Pandey G, Shetty S. Treatment algorithms for managing hepatocellular carcinoma. J Clin Exp Hepatol. 2014;4:S80-S89. doi:10.1016/j.jceh.2014.05.004 
4. Chok KSH, Chan SC, Cheung TT, et al. Late recurrence of hepatocellular carcinoma after liver transplantation. World J Surg. 2011;35:2058-2062. doi:10.1007/s00268-011-1146-Z

5. Okada S, Shimada K, Yamamoto J, et al. Predictive factors for postoperative recurrence of hepatocellular carcinoma. Gastroenterology. 1994;106:1618-1624. doi:10.1016/0016-5085(94)90419-7

6. Kumada T, Nakano S, Takeda I, et al. Patterns of recurrence after initial treatment in patients with small hepatocellular carcinoma. Hepatol Baltim Md. 1997;25:87-92. doi:10.1053/jhep.1997.v25.pm0008985270

7. Portolani N, Coniglio A, Ghidoni S, et al. Early and late recurrence after liver resection for hepatocellular carcinoma: prognostic and therapeutic implications. Ann Surg. 2006;243:229-235. doi:10.1097/ 01.sla.0000197706.21803.a1

8. Llovet JM, Ricci S, Mazzaferro V, et al. Sorafenib in advanced hepatocellular carcinoma. $N$ Engl J Med. 2008;359:378-390. doi:10.1056/NEJMoa0708857

9. Kudo M, Finn RS, Qin S, et al. Lenvatinib versus sorafenib in first-line treatment of patients with unresectable hepatocellular carcinoma: a randomised phase 3 non-inferiority trial. Lancet. 2018;391:1163-1173. doi:10.1016/S0140-6736(18)30207-1

10. Vitale A, Boccagni P, Kertusha X, et al. Sorafenib for the treatment of recurrent hepatocellular carcinoma after liver transplantation? Transplant Proc. 2012;44:1989-1991. doi:10.1016/j.transproceed.2012.06.046

11. Sotiropoulos GC, Nowak KW, Fouzas I, et al. Sorafenib treatment for recurrent hepatocellular carcinoma after liver transplantation. Transplant Proc. 2012;44:2754-2756. doi:10.1016/j.transproceed.2012.09.022

12. Bhoori S, Toffanin S, Sposito C, et al. Personalized molecular targeted therapy in advanced, recurrent hepatocellular carcinoma after liver transplantation: a proof of principle. $J$ Hepatol. 2010;52:771-775. doi:10.1016/j.jhep.2010.01.025

13. Newell P, Toffanin S, Villanueva A, et al. Ras pathway activation in hepatocellular carcinoma and anti-tumoral effect of combined sorafenib and rapamycin in vivo. $J$ Hepatol. 2009;51:725-733. doi:10.1016/j.jhep.2009.03.028

14. Yoon DH, Ryoo B-Y, Ryu M-H, et al. Sorafenib for recurrent hepatocellular carcinoma after liver transplantation. Jpn J Clin Oncol. 2010;40:768-773. doi:10.1093/jjco/hyq055

15. Saab S, McTigue M, Finn RS, Busuttil RW. Sorafenib as adjuvant therapy for high-risk hepatocellular carcinoma in liver transplant recipients: feasibility and efficacy. Exp Clin Transplant Off J Middle East Soc Organ Transplant. 2010;8:307-313.

16. Wang S-N, Chuang S-C, Lee K-T. Efficacy of sorafenib as adjuvant therapy to prevent early recurrence of hepatocellular carcinoma after curative surgery: a pilot study: sorafenib prevents early HCC recurrence. Hepatol Res. 2014;44:523-531. doi:10.1111/hepr.12159

17. Pfiffer TE, Seehofer D, Nicolaou A, et al. Relapse of hepatocellular carcinoma (HCC) after liver transplantation (LT): a single-center analysis on 139 patients in the sorafinib era. J Clin Oncol. 2010;28:4088. doi:10.1200/jco.2010.28.15_suppl.4088

18. Bruix J, Takayama T, Mazzaferro V, et al. Adjuvant sorafenib for hepatocellular carcinoma after resection or ablation (STORM): a phase 3, randomised, double-blind, placebo-controlled trial. Lancet Oncol. 2015;16:1344-1354. doi:10.1016/S1470-2045(15)00198-9

19. Llovet JM, Bruix J. Novel advancements in the management of hepatocellular carcinoma in. $J$ Hepatol. 2008;48:S20-S37. doi:10.1016/j.jhep.2008.01.022

20. Alsina AE, Makris A, Nenos V, et al. Can sorafenib increase survival for recurrent hepatocellular carcinoma after liver transplantation? A pilot study. Am Surg. 2014;80:680-684.

21. Ning Chong CC, San Lai PB. Treatment strategy for recurrent hepatocellular carcinoma. In: Julianov A, editor. Liver Tumors. London: InTech; 2012: Chapter 8.

22. De Simone P, Crocetti L, Pezzati D, et al. Efficacy and safety of combination therapy with everolimus and sorafenib for recurrence of hepatocellular carcinoma after liver transplantation. Transplant Proc. 2014;46:241-244. doi:10.1016/j.transproceed.2013.10.035
23. Takahara T, Nitta H, Hasegawa $Y$, et al. Using sorafenib for recurrent hepatocellular carcinoma after liver transplantation-interactions between calcineurin inhibitor: two case reports. Transplant Proc. 2011;43:2800-2805. doi:10.1016/j.transproceed.2011.06.063

24. Yokoyama I, Carr B, Saitsu H, et al. Accelerated growth rates of recurrent hepatocellular carcinoma after liver transplantation. Cancer. 1991;68:2095-2100.

25. Kim R, Aucejo F. Radiologic complete response with sirolimus and sorafenib in a hepatocellular carcinoma patient who relapsed after orthotopic liver transplantation. $J$ Gastrointest Cancer. 2011;42:50-53. doi:10.1007/s12029-010-9196-2

26. Feun LG, Levi D, Moon J, et al. Tzakis A Sorafenib in hepatocellular carcinoma (HCC) patients after liver transplantation. J Clin Oncol. 2009;27(15):e15579.

27. Shetty K, Dash C, Laurin J. Use of adjuvant sorafenib in liver transplant recipients with high-risk hepatocellular carcinoma. J Transplant. 2014;(2014):913634. doi:10.1155/2014/913634

28. Yokoo H, Kamiyama T, Kakisaka T, et al. [Efficacy of sorafenib for extrahepatic recurrence of hepatocellular carcinoma after liver resection]. Gan To Kagaku Ryoho. 2015;42:1497-1499.

29. Weinmann A, Niederle IM, Koch S, et al. Sorafenib for recurrence of hepatocellular carcinoma after liver transplantation. Dig Liver Dis Off J Ital Soc Gastroenterol Ital Assoc Study Liver. 2012;44:432-437. doi:10.1016/j.dld.2011.12.009

30. Clavien P-A, Lesurtel M, Bossuyt PMM, et al. Recommendations for liver transplantation for hepatocellular carcinoma: an international consensus conference report. Lancet Oncol. 2012;13:e11-e22. doi:10.1016/S1470-2045(11)70175-9

31. Kim HY, Park J-W, Nam B-H, et al. Survival of patients with advanced hepatocellular carcinoma: sorafenib versus other treatments. J Gastroenterol Hepatol. 2011;26:1612-1618. doi:10.1111/j.14401746.2011.06751.x

32. Sposito C, Mariani L, Germini A, et al. Comparative efficacy of sorafenib versus best supportive care in recurrent hepatocellular carcinoma after liver transplantation: a case-control study. $J$ Hepatol. 2013;59:59-66. doi:10.1016/j.jhep.2013.02.026

33. Zhang W, Zhao G, Wei K, et al. Adjuvant sorafenib reduced mortality and prolonged overall survival and post-recurrence survival in hepatocellular carcinoma patients after curative resection: a single-center experience. Biosci Trends. 2014;8:333-338. doi:10.5582/bst.2014.01120

34. Liu L, Cao Y, Chen C, et al. Sorafenib blocks the RAF/MEK/ERK pathway, inhibits tumor angiogenesis, and induces tumor cell apoptosis in hepatocellular carcinoma model PLC/PRF/5. Cancer Res. 2006;66:11851-11858. doi:10.1158/0008-5472.CAN-06-1377

35. Wei J, Meng F, Qu K, et al. Sorafenib inhibits proliferation and invasion of human hepatocellular carcinoma cells via up-regulation of p53 and suppressing FoxM1. Acta Pharmacol Sin. 2015;36:241-251. doi:10.1038/aps.2014.122

36. Furuse J. Sorafenib for the treatment of unresectable hepatocellular carcinoma. Biol Targets Ther. 2008;2:779-788. doi:10.2147/BTT.S3410

37. Heqing Y, Bin L, Xuemei Y, Linfa L. The role and mechanism of autophagy in sorafenib targeted cancer therapy. Crit Rev Oncol Hematol. 2016;100:137-140. doi:10.1016/j.critrevonc.2016.02.006

38. Kitade H, Yanagida H, Yokoigawa N, et al. [Two patients with recurrence of hepatocellular carcinoma after liver resection who achieved long-term stable disease with small doses of sorafenib therapy]. Gan To Kagaku Ryoho. 2015;42:863-865.

39. Feng Y-X, Wang T, Deng Y-Z, et al. Sorafenib suppresses postsurgical recurrence and metastasis of hepatocellular carcinoma in an orthotopic mouse model. Hepatol Baltim Md. 2011;53:483-492. doi:10.1002/hep.24075 


\section{Supplementary materials}

Table SI Case distribution

\begin{tabular}{|c|c|c|c|}
\hline Center & Group & No. in the group & No. rejected \\
\hline \multirow[t]{3}{*}{ Xijing Hospital } & Sorafenib & 13 & 0 \\
\hline & Control group & 24 & 0 \\
\hline & Total & 37 & 0 \\
\hline \multirow[t]{3}{*}{ Anhui Provincial Hospital } & Sorafenib & 11 & 2 \\
\hline & Control group & 22 & 0 \\
\hline & Total & 33 & 2 \\
\hline \multirow[t]{3}{*}{ Sichuan Huaxi Hospital } & Sorafenib & 13 & 1 \\
\hline & Control group & 8 & 0 \\
\hline & Total & 21 & 1 \\
\hline \multirow[t]{3}{*}{ Sichuan tumor } & Sorafenib & 2 & 0 \\
\hline & Control group & 3 & 0 \\
\hline & Total & 5 & 0 \\
\hline \multirow[t]{3}{*}{ Southwest Hospital } & Sorafenib & 10 & 1 \\
\hline & Control group & 10 & 0 \\
\hline & Total & 20 & I \\
\hline \multirow[t]{3}{*}{ Guangxi Medical University's Tumor Hospital } & Sorafenib & 4 & 0 \\
\hline & Control group & 7 & 2 \\
\hline & Total & 11 & 2 \\
\hline \multirow[t]{3}{*}{ Guangxi Medical University's First Affiliated Hospital } & Sorafenib & 5 & 3 \\
\hline & Control group & 5 & 5 \\
\hline & Total & 10 & 8 \\
\hline \multirow[t]{3}{*}{ First Xiangya Hospital } & Sorafenib & 11 & 0 \\
\hline & Control group & 11 & 0 \\
\hline & Total & 22 & 0 \\
\hline \multirow[t]{3}{*}{ Second Xiangya Hospital } & Sorafenib & 4 & 2 \\
\hline & Control group & I & 0 \\
\hline & Total & 5 & 2 \\
\hline \multirow[t]{3}{*}{ Henan Tumor Hospital } & Sorafenib & 7 & 0 \\
\hline & Control group & 7 & 0 \\
\hline & Total & 14 & 0 \\
\hline \multirow[t]{3}{*}{ Kunming Medical University's First Hospital } & Sorafenib & 4 & 0 \\
\hline & Control group & 4 & 0 \\
\hline & Total & 8 & 0 \\
\hline \multirow[t]{3}{*}{ Xijiang Tumor Hospital } & Sorafenib & 12 & 0 \\
\hline & Control group & 6 & 0 \\
\hline & Total & 18 & 0 \\
\hline \multirow[t]{3}{*}{ Wuhan Union Hospital } & Sorafenib & I & 0 \\
\hline & Control group & 6 & 0 \\
\hline & Total & 7 & 0 \\
\hline \multirow[t]{3}{*}{ Wuhan Tongji Hospital } & Sorafenib & 6 & 0 \\
\hline & Control group & 0 & 0 \\
\hline & Total & 6 & 0 \\
\hline \multirow[t]{3}{*}{ Jiangxi Tumor Hospital } & Sorafenib & 4 & 0 \\
\hline & Control group & 4 & 0 \\
\hline & Total & 8 & 0 \\
\hline \multirow[t]{3}{*}{ Total } & Sorafenib & 107 & 9 \\
\hline & Control group & 118 & 7 \\
\hline & Total & 225 & 16 \\
\hline
\end{tabular}


Table S2 Preoperative tumor load

\begin{tabular}{|c|c|c|c|c|c|}
\hline Items & Indicators & Sorafenib & Control & Total & $P$-value \\
\hline \multirow[t]{3}{*}{ Tumor position I } & $0, \mathrm{n}(\%)$ & $3(3.19)$ & $3(3.23)$ & $6(3.17)$ & 0.431 \\
\hline & Selected, n (\%) & $6(6.38)$ & $2(2.15)$ & $8(4.23)$ & \\
\hline & Not selected, n (\%) & $85(90.43)$ & $88(94.62)$ & 175 (92.59) & \\
\hline \multirow[t]{2}{*}{ Tumor position II } & Selected, n (\%) & $18(19.78)$ & $22(24.44)$ & $4 \mathrm{I}(22.40)$ & 0.450 \\
\hline & Not selected, n (\%) & $73(80.22)$ & $68(75.56)$ & $142(77.60)$ & \\
\hline \multirow[t]{2}{*}{ Tumor position III } & Selected, n (\%) & $16(17.58)$ & $23(25.56)$ & $40(21.86)$ & 0.192 \\
\hline & Not selected, n (\%) & $75(82.42)$ & $67(74.44)$ & 143 (78.14) & \\
\hline \multirow[t]{2}{*}{ Tumor position IV } & Selected, n (\%) & $18(19.78)$ & $24(26.67)$ & $43(23.50)$ & 0.272 \\
\hline & Not selected, n (\%) & $73(80.22)$ & $66(73.33)$ & $140(76.50)$ & \\
\hline \multirow[t]{2}{*}{ Tumor position $\mathrm{V}$} & Selected, n (\%) & $37(40.66)$ & $42(46.67)$ & $80(43.72)$ & 0.415 \\
\hline & Not selected, n (\%) & $54(59.34)$ & $48(53.33)$ & $103(56.28)$ & \\
\hline \multirow[t]{2}{*}{ Tumor position VI } & Selected, n (\%) & $47(51.65)$ & 51 (56.67) & $99(54.10)$ & 0.498 \\
\hline & Not selected, n (\%) & $44(48.35)$ & $39(43.33)$ & $84(45.90)$ & \\
\hline \multirow[t]{2}{*}{ Tumor position VII } & Selected, n (\%) & $4 I(45.05)$ & $33(36.67)$ & 75 (40.98) & 0.251 \\
\hline & Not selected, n (\%) & $50(54.95)$ & $57(63.33)$ & 108 (59.02) & \\
\hline \multirow[t]{2}{*}{ Tumor position VIII } & Selected, n (\%) & $33(36.26)$ & $25(27.78)$ & $59(32.24)$ & 0.221 \\
\hline & Not selected, n (\%) & $58(63.74)$ & $65(72.22)$ & $124(67.76)$ & \\
\hline \multirow[t]{5}{*}{ Number of focuses } & $0, \mathrm{n}(\%)$ & $0(0.00)$ & $2(1.74)$ & $2(0.89)$ & 0.672 \\
\hline & I, n (\%) & $76(70.37)$ & $83(72.17)$ & $159(70.67)$ & \\
\hline & $2, \mathrm{n}(\%)$ & 15 (I3.89) & II (9.57) & $27(12.00)$ & \\
\hline & $3, \mathrm{n}(\%)$ & $5(4.63)$ & $5(4.35)$ & $10(4.44)$ & \\
\hline & $>3, \mathrm{n}(\%)$ & $12(I 1 . I I)$ & $14(12.17)$ & $27(12.00)$ & \\
\hline \multirow[t]{2}{*}{ Maximum diameter } & $\mathrm{N}$ (missing) & $108(0)$ & $114(1)$ & $224(I)$ & 0.145 \\
\hline & Mean (SD) & $7.90(3.48)$ & $7.30(3.37)$ & $7.55(3.44)$ & \\
\hline \multirow[t]{2}{*}{ Sum of long diameter } & $\mathrm{N}$ (missing) & $108(0)$ & III (4) & $221(4)$ & 0.031 \\
\hline & Mean (SD) & $9.25(4.20)$ & $8.66(5.19)$ & $8.93(4.72)$ & \\
\hline \multirow[t]{2}{*}{ Blood vessel and bile duct invasion } & None, n (\%) & $60(56.60)$ & $63(55.26)$ & $124(55.86)$ & 0.841 \\
\hline & Existing, n (\%) & $46(43.40)$ & $51(44.74)$ & $98(44.14)$ & \\
\hline \multirow[t]{4}{*}{ Invaded position } & Portal vein, n (\%) & $39(84.78)$ & $35(71.43)$ & $74(77.08)$ & 0.196 \\
\hline & Hepatic vein, n (\%) & $2(4.35)$ & $4(8.16)$ & $6(6.25)$ & \\
\hline & Capillaries, n (\%) & $4(8.70)$ & $10(20.4 I)$ & $15(15.63)$ & \\
\hline & Bile duct, n (\%) & I (2.17) & $0(0.00)$ & I (I.04) & \\
\hline \multirow[t]{2}{*}{ Lymphatic metastasis } & None, n (\%) & $105(98.13)$ & I I 2 (98.25) & $219(98.21)$ & 1.000 \\
\hline & Existing, n (\%) & $2(1.87)$ & $2(1.75)$ & $4(1.79)$ & \\
\hline \multirow[t]{2}{*}{ Distant metastasis } & None, n (\%) & I0I (94.39) & $113(99.12)$ & $216(96.86)$ & 0.059 \\
\hline & Existing, n (\%) & $6(5.6 I)$ & I (0.88) & $7(3.14)$ & \\
\hline \multirow[t]{2}{*}{ Distant metastasis positions } & Lung, n (\%) & I (33.33) & $0(0.00)$ & I (33.33) & $<0.001$ \\
\hline & Others, n (\%) & $2(66.67)$ & $0(0.00)$ & $2(66.67)$ & \\
\hline
\end{tabular}

OncoTargets and Therapy

\section{Publish your work in this journal}

OncoTargets and Therapy is an international, peer-reviewed, open access journal focusing on the pathological basis of all cancers, potential targets for therapy and treatment protocols employed to improve the management of cancer patients. The journal also focuses on the impact of management programs and new therapeutic agents and protocols on patient perspectives such as quality of life, adherence and satisfaction. The manuscript management system is completely online and includes a very quick and fair peer-review system, which is all easy to use. Visit http://www.dovepress.com/ testimonials.php to read real quotes from published authors. 